\title{
D2-D8 system with massive strings and the Lifshitz spacetimes
}

\author{
Harvendra Singh \\ Theory Division, Saha Institute of Nuclear Physics, \\ 1/AF, Bidhannagar, Kolkata 700064, India \\ Homi Bhabha National Institute, \\ Anushakti Nagar, Mumbai 400094, India \\ E-mail: h.singh@saha.ac.in
}

ABSTRACT: The Romans' type IIA supergravity allows fundamental strings to have explicit mass term at the tree level. We show that there exists a (F1,D2,D8) brane configuration which gives rise to $L i f_{4}^{(2)} \times R^{1} \times S^{5}$ vacua supported by the massive strings. The presence of D8-branes naturally excites massive fundamental strings. A compactification on circle relates these Lifshitz massive type-IIA background with the axion-flux $L i f_{4}^{(2)} \times S^{1} \times S^{5}$ vacua in ordinary type-IIB theory. The massive T-duality in eight dimensions further relates them to yet another $\widetilde{\mathrm{Lif}_{4}^{(2)}} \times S^{1} \times S^{5}$ vacua constituted by (F1,D0,D6) system in ordinary type IIA theory. The latter vacua after compactification to four dimensions generate two 'distinct' electric charges and a constant magnetic field, all living over 2-dimensional plane. This somewhat reminds us of a similar set up in quantum Hall systems.

KEYwords: D-branes, AdS-CFT Correspondence, Flux compactifications, String Duality ARXIV EPRINT: 1701.00968 


\section{Contents}

1 Introduction 1

$2 \mathrm{Sch}_{4}^{(3)} \times S^{6}$ : massive strings $\quad 2$

$3 \operatorname{Lif}_{4}^{(2)} \times R^{1} \times S^{5}$ vacua $\quad 4$

3.1 D2-D8: tied up with massive strings 4

$3.2 \quad$ Lif $_{4}^{(2)} \times S_{w}^{1} \times S^{5} \quad 5$

3.3 Massive $9 d$ T-duality 5

$\begin{array}{ll}3.45 d \text { massive tensor model } & 7\end{array}$

3.5 The $4 d$ Proca model 8

$4 T^{2}$ dual of $(F 1, D 2, D 8)$ system $\quad 8$

5 Lower dimensional models $\quad 9$

$5.14 d$ Lifshitz theory and a quantum Hall system 9

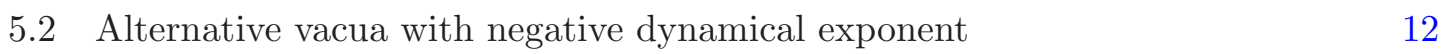

$\begin{array}{lll}5.3 & 5 d \text { vortex model: } B \wedge G \text { interaction } & 13\end{array}$

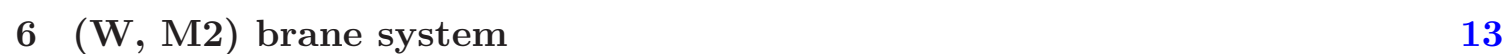

$\begin{array}{lll}7 & \text { Summary } & 14\end{array}$

\section{Introduction}

The AdS/CFT [1-3] holgraphic applications in strongly coupled quantum systems exhibiting nonrelativitic symmetries near critical points has been the focus of studies recently [4][30]. Some of the holographic applications may involve strongly coupled fermionic systems at finite density or a gas of ultra cold atoms $[4,5]$. Some related framework of Galilean symmetry were studied even earlier [21, 22]. For finite temperature properties such as phase transitions, transport and viscosity we include black-holes in asymptotically AdS backgrounds. For superconductivity phenomenon the 4-dimensional non-relativistic geometry generically involves spontaneously broken Higgs phase where the Maxwell field is massive $[6,10]$. We shall discuss here some examples of 10-dimensional Lifshitz spacetimes where a Higgs phase instead involves 2-rank antisymmetric tensor field and has massive fundamental strings. The two phenomena indeed have parallel from 10-dimensional perspective. It is because a Kaluza-Klein compactification of a (massive) 2-rank tensor field on a circle gives rise to a (massive) gauge field in lower dimensions.

Our main interest in this work is to construct Lifshitz solutions with dynamical exponent $a=2$, directly in Roman's type IIA supergravity [31]. The massive type IIA theory is 
the only known example of a 10-dimensional maximal supergravity where the string field is explicitly massive at the tree level. Thus the massive type IIA provides an unique setup to look for Lifshitz and Schrödinger like solutions which involve massive fields and study their dual nonrelativistic field theories on the boundary. There are no prior attempts to our knowledge where the same has been worked out for Romans type IIA supergravity, however $S c h_{4}^{(3)} \times S^{6}$ massive string vacua are known to us [23]. The $L i f_{4}^{(2)} \times R^{1} \times S^{5}$ have been shown to exist in $10 d$ type IIB string theory when appropriate axion flux is switched on [25]. We provide an exlicit example of $L i f_{4}^{(2)} \times R^{1} \times S^{5}$ background, which is a solution of massive type IIA and it does involve massive $B$-field. It is a background generated by a bound state of $(F 1, D 2, D 8)$ brane system. It is important to know all such vacua as their compactification on $S^{1} \times S^{5}$ immediately provides the prototype $L i f_{4}^{(2)}$ background in $4 d$, which is proposed to be dual to non-relativistic Lifshitz theory with dynamical exponent two. By exploiting massive T-duality symmetries in $8 d$ we further provide another example of $\widetilde{\operatorname{Lif}_{4}^{(2)}} \times S^{1} \times S^{5}$ background, which is generated by massless $(F 1, D 0, D 6)$ brane system in ordinary type IIA theory.

The paper is organised as follows. In the section- 2 we first review the relevant aspects of massive type IIA sugra action and its known Schrödinger solution. In section-3 we write down new Lifshitz solution generated by the $(F 1, D 2, D 8)$ system and discuss subtle aspects under massive T-duality for these solutions. The section- 4 contains ordinary type IIA solution involving $(F, D 0, D 6)$ branes, which is supported by RR 2 -form flux and massless $B$-field. They have a very crucial $\left(B_{(2)} \wedge G_{(2)}\right)^{2}$ coupling between them. The string $B$-field gets essentially nested with constant magnetic flux of $G_{(2)}$ over $2 d$ plane. We next discuss their consistent reduction to four and five dimensions and their effective bulk theories in section-5. We provide an M-theory uplift of these vacua in section-6. A summary is provided in the section- 7 .

\section{$2 \operatorname{Sch}_{4}^{(3)} \times S^{6}:$ massive strings}

In this section we mainly review some useful information about 10-dimensional massive type IIA supergravity [31] and a known Schrödinger vacua $S_{c h}^{(3)} \times S^{6}$ [23]. One may choose to directly skip to the next section. The Romans type IIA theory is the only known maximal supergravity in ten dimensions which allows massive string $B$ field. The theory is described by the following bosonic action

$$
S=\frac{1}{G_{N}} \int\left[e^{-2 \phi}\left(* R+4(d \phi)^{2}-\frac{1}{2}\left(H_{(3)}\right)^{2}\right)-\frac{1}{2}\left(G_{(2)}\right)^{2}-\frac{1}{2}\left(G_{(4)}\right)^{2}-* \frac{m^{2}}{2}\right]
$$

where topological terms have been dropped because these would be vanishing for the Lifshitz backgrounds we shall be studying in this paper, see for details in [31, 39]. ${ }^{1}$ The field strengths are defined as

$$
H_{(3)}=d B_{(2)}, \quad G_{(2)}=d C_{(1)}+m B_{(2)}, \quad G_{(4)}=d C_{(3)}+B_{(2)} \wedge d C_{(1)}+\frac{m}{2} B_{(2)} \wedge B_{(2)}
$$

\footnotetext{
${ }^{1}$ We are adopting a convention: $\int\left(H_{(p)}\right)^{2}=\int H_{(p)} \wedge * H_{(p)}=\frac{1}{p !} \int d^{10} x \sqrt{-g} H_{\mu_{1} \cdots \mu_{p}} H^{\mu_{1} \cdots \mu_{p}}$ and for scalar quantities like curvature scalar: $\int * R=\int d^{10} x \sqrt{-g} R$.
} 
where $m$ is the mass parameter and positive cosmological constant of the 10-dimensional theory. The cosmological constant however generates a nontrivial potential term for the dilaton field. Other than the nonsupersymmetric Freund-Rubin vacua in [31], some known BPS solutions of the theory include D8-branes [32-37], the $K 3$ compactifications [38], the $(D 6, D 8)$ and $(D 4, D 6, D 8)$ bound states $[39,40]$. Under the 'massive' T-duality [33] the D8 brane can be mapped over to a D7 brane in ordinary type IIB string theory. The string $B$-field is explicitly massive with mass square given by $m^{2}$, and it plays an important role in obtaining nonrelativistic Schrödinger solutions [23]. The massive type IIA theory however never admits a flat Minkowski vacuum solution. But in the $m \rightarrow 0$ limit the massive theory reduces to ordinary type IIA supergravity.

An observed common feature in four-dimensional AdS gravity theories has been that in order to obtain Schrödinger or Lifshitz type non-relativistic solutions one needs to include massive (Proca) gauge fields in the action [4, 5]. (Although massless gauge fields can give rise to nonrelativistic vacua however, in simple cases of D-branes compactified along lightcone coordinate [26, 27], they usually give rise to conformal (or hyperscaling) Lifshitz or Schrödinger vacua.) Particularly for massive type IIA theory the existence of Schrödinger solution $S c h_{4}^{(3)} \times S^{6}$ has already been shown in [23]. In the rest of this section we review the $a=3$ Schrödinger solution to familiarise ourselves, and also because these vacua are constituted by D0, D2, D8 branes, alongwith massive strings which we will encounter again when we write down Lifshitz solutions of the theory.

The massive type IIA action in the tensorial notation is given by

$S=\frac{1}{G_{N}} \int d^{10} x \sqrt{-g}\left[R-\frac{1}{2}\left(\partial_{\mu} \phi\right)^{2}-\frac{e^{-\phi}}{2.3 !}\left(H_{\mu \nu \lambda}\right)^{2}-\frac{e^{3 \phi / 2}}{2.2 !}\left(G_{\mu \nu}\right)^{2}-\frac{e^{\phi / 2}}{2.4 !}\left(G_{\mu \nu \lambda \sigma}\right)^{2}-\frac{e^{5 \phi / 2}}{2} m^{2}\right]$,

with the field strengths $H_{\mu \nu \lambda}=3 \partial_{[\mu} B_{\nu \lambda]}, G_{\mu \nu}=2 \partial_{[\mu} C_{\nu]}+m B_{\mu \nu}, G_{\mu \nu \lambda \sigma}=4 \partial_{[\mu} C_{\nu \lambda \sigma]}+$ $6 B_{[\mu \nu} G_{\nu \sigma]}-3 m B_{[\mu \nu} B_{\nu \sigma]}$. The equations of motion of this theory admit following $S c c_{4}^{(3)} \times S^{6}$ solution $[23]$

$$
\begin{aligned}
d s^{2} & =L^{2}\left(-\frac{2}{z^{6}}\left(d x^{+}\right)^{2}+\frac{-2 d x^{+} d x^{-}+d y^{2}+d z^{2}}{z^{2}}+\frac{5}{2} d \Omega_{6}^{2}\right), \\
e^{\phi} & =g_{a}, \quad C_{+-y}=-\sqrt{10} g_{a}^{-\frac{1}{4}} \frac{L^{3}}{3 z^{3}}, \quad C_{+}=-\frac{\sqrt{5}}{3} \frac{g_{a}^{-3 / 4} L}{z^{3}} \\
B_{+y} & =-\frac{g_{a} L^{2}}{2 z^{4}}
\end{aligned}
$$

where $L^{2}=\frac{2}{m^{2} g_{a}^{5 / 2}}$. But we should have string coupling $g_{a} \ll 1$ and $L \gg 1$. The radius of curvature of the sphere is directly related to the mass parameter. Note that the Lorentz invariance is explicitly broken in the solutions, although spin group of the sphere is intact. The Schrödinger vacua (2.4) involves a collection of D0, D2, and D8 branes (which are wrapped around $S^{6}$ ), along with massive fundamental strings (F1) stretched along $y$ direction. The Einstein equations do involve a nontrivial stress-energy tensor component $T_{++}$ that receives contributions from the lightlike components of these fields. From the solution we learn that in ten-dimensional sense the matter (dust) responsible for a Schödinger solutions is made up of D-branes and most importantly the massive strings. The boundary of 
the spacetime is located at $z=0$, and near to the boundary the metric and fields become divergent, which usually is the case for non-relativistic vacua with $a>1$. But everything is fine in the interior of the spacetime including the curvature scalar which is a constant quantity.

\section{$3 \quad \operatorname{Lif}_{4}^{(2)} \times R^{1} \times S^{5}$ vacua}

\subsection{D2-D8: tied up with massive strings}

It is known that the Freund-Rubin $A d S_{4} \times S^{6}$ maximally symmetric vacua in Romans theory [31] is constituted by $(D 2, D 8)$ branes. But there are no 'massive' $B$-field excitations in them. We show that one can construct Lifshitz vacua that are supported by massive $B$-field, D2 and D8 branes. The strings in these solutions becomes massive after gobbling up all D0 branes, unlike the Schrödinger type vacua (2.4) above where some D0 branes are still present. Thus the D0 brane charges will not be explicitly seen in the following Lifshitz solutions. These higgsed Lifshitz solutions are given by (in string metric)

$$
\begin{aligned}
d s^{2} & =\alpha^{\prime} L^{2}\left(-\frac{d t^{2}}{z^{4}}+\frac{d x_{1}^{2}+d x_{2}^{2}}{z^{2}}+\frac{d z^{2}}{z^{2}}+\frac{d y^{2}}{q^{2}}+d \Omega_{5}^{2}\right), \\
e^{\phi} & =g_{a}, \quad C_{(3)}=-\frac{\left(\alpha^{\prime} L^{2}\right)^{\frac{3}{2}}}{g_{a}} \frac{1}{z^{4}} d t \wedge d x_{1} \wedge d x_{2}, \\
B_{(2)} & =\frac{\alpha^{\prime} L^{2}}{q z^{2}} d t \wedge d y,
\end{aligned}
$$

with $L=\frac{2}{g_{a} m l_{s}}$, where $m$ being the mass parameter in the Romans' action. The $q$ is free (length) parameter and $g_{a}$ is perturbative string coupling in this massive type IIA vacuum. Note $L$, which is dimensionless, determines overall radius of curvature of the 10-dimensional spacetime. While $m$ being a parameter in the lagrangian and it determines $L$, therefore Romans' theory with $m \ll \frac{2}{g_{a} l_{s}}$ would be preferred so that we can have $L \gg 1$ in the solutions (3.1), else we cannot trust these classical vacua. ${ }^{2}$

The Lifshitz configuration (3.1) describes a parallel stack of D2-branes stretched along $\left(x_{1}, x_{2}\right)$ directions and 'massive' fundamental strings that are aligned along $y$ direction. The D8-branes wrap around $S^{5}$ completely while remaining stretched along the patch $\left(x_{1}, x_{2}, y\right)$. The D2 charges can be found as

$$
Q_{D 2} \sim \frac{1}{\omega_{5}} \int e^{\frac{\phi}{2}} \star G_{(4)} \sim \frac{4 l_{s}^{5} L^{5} l_{y}}{g_{a} q} \propto N
$$

where $l_{y}$ is coordinate size of $y$ and $\omega_{5}$ is the unit volume of 5-sphere. Thus eq.(3.1) describes a $(F 1, D 2, D 8)$ configuration, in which D2 branes are stacked inside D8 worldvolume. The D2 stack is studded (threaded) by massive F-strings, having mass $m=\frac{2}{L g_{a} l_{s}}$. The D0 branes are all gauged away (or eaten up) by the F-strings which have become massive. This phenomenon happens due to the higgsing (or stueckelberg mechanism) in massive

\footnotetext{
${ }^{2}$ Note, from the D8 brane/domain wall correspondence [33], one typically expects $m \approx \frac{g_{a} N_{D 8}}{l_{s}}$, a value which is definitely well within $\frac{2}{g_{a} l_{s}}$.
} 
type IIA theory [31]. In these solutions $y$ remains an overall isometry direction which may be compact also. The holographic $z$ coordinate acts as a common transverse direction for all the branes.

\section{$3.2 \quad \operatorname{Lif}_{4}^{(2)} \times S_{w}^{1} \times S^{5}$}

The 10-dimensional string coupling $g_{a}$ has to be weak so that we can trust the Lifshitz vacua (3.1). The small $g_{a}$ however tends to send the mass of F-strings to higher values, but for sufficiently large $L \sim O\left(\frac{1}{g_{a}^{2}}\right)$ it can support massive strings in the background (note the lighter ones with $m \approx \frac{g_{a}}{l_{s}}$ only need to be excited). So far we considered $y$ as noncompact coordinate and $q$ an associated arbitary length scale. Let us consider the case when $y$ is a compact circle, i.e. $y \sim y+2 \pi R_{y}$. The physical radius of $y$-circle will be $\frac{l_{s} L R_{y}}{q}$. Therefore for smaller $q$ values the massive strings can indeed be excited in the transverse $y$ direction of the D2-branes. It would also be appropriate here to take $q \equiv w R_{y}$, with $w$ being an integer. (It could also be the wrapping number of D8-branes. By wrapping number here we mean by number of times a single D8 brane wraps around $S^{1}$.) The vacua (3.1) will now be described as $\operatorname{Lif} f_{4}^{(2)} \times S_{w}^{1} \times S^{5}$ :

$$
\begin{aligned}
d s^{2} & =\alpha^{\prime} L^{2}\left(-\frac{d t^{2}}{z^{4}}+\frac{d x_{1}^{2}+d x_{2}^{2}}{z^{2}}+\frac{d z^{2}}{z^{2}}+\frac{1}{w^{2}} d \psi^{2}+d \Omega_{5}^{2}\right) \\
e^{\phi} & =g_{a}, \quad C_{(3)}=-\frac{\left(\alpha^{\prime} L^{2}\right)^{\frac{3}{2}}}{g_{a} z^{4}} d t \wedge d x_{1} \wedge d x_{2}, \quad B_{(2)}=\frac{\alpha^{\prime} L^{2}}{w z^{2}} d t \wedge d \psi,
\end{aligned}
$$

where $0 \leq \psi \leq 2 \pi$. ( $\psi$ can also be viewed as an orbifolded circle.) Anyhow $w>1$ tells us about the comparative sizes of $S^{1}$ and $S^{5}$. Due to this size difference a D8-brane can wrap $S^{1} w$-times more as compared to its wrapping around $S^{5}$. Especially for $\omega=1$, both $S^{1}$ and $S^{5}$ will have the same size. But small $w$ is always preferred in (3.3). For large $w$ (or $q$ ) the radius of $S^{1}$ will become sub-stringy, it would then be appropriate to switch over to the T-dual vacua in type IIB string theory. We would discuss it in the next section.

The Lifshitz solutions (3.1) or (3.3) both have following asymmetric scaling symmetry

$$
t \rightarrow \lambda^{2} t, \quad x^{i} \rightarrow \lambda x^{i}, \quad z \rightarrow \lambda z
$$

Note $y($ or $\psi)$ coordinate is not required to scale at all and it is one of the charactersitics of the $a=2$ Lifshitz vacua.

\subsection{Massive 9d T-duality}

Upon $S^{1}$ compactification the Romans theory gives rise to a massive supergravity in nine dimensions. Keeping only the fields relevant for our background (3.1), (a complete circle compactification can be found [33]) a $9 d$ massive action can be written as

$$
\begin{gathered}
S \sim \int d^{9} x \sqrt{-g} e^{-2 \phi_{9}}\left[R+4\left(\partial_{\mu} \phi_{9}\right)^{2}-\frac{1}{2}\left(\partial_{\mu} \rho\right)^{2}-\frac{e^{\sqrt{2} \rho}}{2.2 !}\left(F_{\mu \nu}\right)^{2}-\frac{e^{\frac{\rho}{\sqrt{2}}}}{2} m^{2}\left(A_{\mu}\right)^{2}\right. \\
\left.-\frac{e^{\frac{-\rho}{\sqrt{2}}}}{2.4 !}\left(G_{\mu \nu \lambda \sigma}\right)^{2}-\frac{e^{\frac{-\rho}{\sqrt{2}}}}{2} m^{2}\right]
\end{gathered}
$$


where the vector field $A_{\mu}$ which arises directly from reduction of massive tensor component $\hat{B}_{\mu \underline{y}}$ is only kept in the action. The $9 d$ dilaton field is given by

$$
\phi_{9}=\hat{\phi}-\frac{1}{2 \sqrt{2}} \rho
$$

whereas

$$
\rho=\frac{1}{\sqrt{2}} \log \left(\hat{g}_{y y}\right)
$$

defines the radion mode along $y$. (The $10 d$ fields are denoted with a hat sign to distinguish them from 9-dimensional ones.) The Kaluza-Klein gauge field is set to vanish. The topological terms in the action are also ignored as these are not relevant for the Lifshitz background we are studying here. By setting $\alpha^{\prime}=1$, after compactification along $y$ the eq.(3.1) reduces to

$$
\begin{aligned}
d s_{9 d}^{2} & =L^{2}\left(-\frac{d t^{2}}{z^{4}}+\frac{d x_{1}^{2}+d x_{2}^{2}+d z^{2}}{z^{2}}+d \Omega_{5}^{2}\right), \\
e^{\phi_{9}} & =\sqrt{\frac{q}{L}} g_{a}, \quad C_{(3)}=-\frac{L^{3}}{g_{a} z^{4}} d t \wedge d x_{1} \wedge d x_{2} \\
A_{(1)} & =\frac{L^{2}}{q z^{2}} d t, \quad e^{-\sqrt{2} \rho}=q^{2} L^{-2}
\end{aligned}
$$

It can be checked that (3.6) is a consistent solution of the $9 d$ action (3.5). Actually both the dilaton $\phi_{9}$ and the $\rho$ have constant background values. Since it is a solution of $9 d$ type II sugra with a massive vector field, the above $9 d$ Lifshitz vacua can be uplifted back to 10-dimensional type IIB theory (over a dual radius circle) by exploiting 'massive' Tduality [33]. By employing the massive duality rules [33, 41] we get following type IIB solution

$$
\begin{aligned}
d s_{I I b}^{2} & =L^{2}\left(\tilde{q}^{2} d \tilde{y}^{2}-\frac{2 \tilde{q} d t d \tilde{y}}{z^{2}}+\frac{d x_{1}^{2}+d x_{2}^{2}}{z^{2}}+\frac{d z^{2}}{z^{2}}+d \Omega_{5}^{2}\right) \\
G_{(5)} & =\frac{1}{\sqrt{2} g_{b}}\left(\frac{4 \tilde{q} L^{4}}{z^{5}} d t \wedge d x_{1} \wedge d x_{2} \wedge d \tilde{y} \wedge d z+\text { self dual }\right) \\
e^{\phi_{b}} & =g_{b}, \quad \chi=\frac{2 \tilde{q}}{g_{b}} \tilde{y} .
\end{aligned}
$$

The type IIB string coupling $g_{b}$, radius of curvature $L$, and flux $\tilde{q}$ are all independent parameters. The coupling $g_{b}$ should be kept small. The parameter $\tilde{q}$ determines the required axion flux and also the relative size of $\tilde{y}$ in the metric. (Recall an ordinary type IIB theory has no mass scale of its own unlike the Romans' type IIA theory.) The massive $9 d$ T-duality does indeed relate $\tilde{q}$ with Romans' mass through

$$
g_{b}=g_{a} \frac{q}{L}, \quad m=\frac{2}{g_{a} L} \equiv \frac{2 \tilde{q}}{g_{b}}, \quad \tilde{q} \equiv \frac{q}{L^{2}}
$$

Note the flux parameter $\tilde{q}$ has mass dimension one. The vacua (3.7) describes Lifshitz solution of type IIB theory first obtained by [25], if we redefine the coordinates as $\tilde{y}=$ $x^{+}, t=x^{-}$. 
Note that the axion field has a linear dependence on the circle coordinate $(\tilde{y} \sim \tilde{y}+$ $2 \pi \tilde{R}_{y}$ ), where $\tilde{R}_{y}=\frac{\alpha^{\prime} L^{2}}{R_{y}}$ via T-duality. Due to this the axion undergoes discrete jumps (determined by parameter $\tilde{q}$ ) each time it goes around the circle, as it has to be periodic. One should take $\tilde{q}=\frac{w R_{y}}{L^{2}}$ with an integral $w$. The $w$ will now effectively count the axions (or the number of D7 branes)

$$
Q_{D 7}=\frac{1}{2 \pi} \int d \chi=\tilde{q} \tilde{R}_{y}=w .
$$

The D3 charge is given by

$$
Q_{D 3} \sim \frac{1}{\omega_{5}} \int \star G_{(5)}=\frac{2 \sqrt{2} L^{4}}{g_{b}} \approx N
$$

taking $L^{4} \approx g_{b} N$. Thus the eq.(3.7) describes a bound state of $(W, D 3, D 7)$ system with wave. The coordinates $z$ and $\tilde{y}$ together constitute two transverse directions of D7 branes. It is the same kind of effect which the massive T-duality brings under which D8-brane of massive type IIA is mapped over to D7-brane in type IIB and vice-versa [33]. Thus we have confirmed that the Romans type IIA theory with massive $B$-field admits $(F 1, D 2, D 8)$ Lifshitz vacua and it also provides a consistent T-dual description of the $(W, D 3, D 7)$ Lifshitz vacua of type IIB string theory.

The vacua presented here are different from other Lifshitz vacua obtained in $6 d$ and $5 d$ gauged/massive supergravities with arbitrary dynamical exponents in [28, 29]. Those $L_{i f}^{(a)} \times H_{2}$ and $\operatorname{Lif}_{3}^{(a)} \times H_{2}$ vacua with $a>1$ (the $H_{2}$ being sphere, hyperboloid or flat space) can also be lifted to $10 d$, but these are different cases. Only dilatonic scalars are present in those gauged/massive sugra theories. We are note sure if they could be ralated to $(F 1, D 2, D 8)$ background.

\section{$3.45 d$ massive tensor model}

After doing an explicit $S^{5}$ compactification the 10-dimensional background (3.1) reduces to the following 5 -dimensional $\operatorname{Lif}_{4}^{(2)} \times R^{1}$ solution (we set $\alpha^{\prime}=1$ for simplicity)

$$
\begin{aligned}
& d s^{2}=L^{2}\left(-\frac{d t^{2}}{z^{4}}+\frac{d x_{1}^{2}+d x_{2}^{2}}{z^{2}}+\frac{d z^{2}}{z^{2}}+\frac{d y^{2}}{q^{2}}\right) \\
& e^{\phi_{5}}=g_{5}, \quad \bar{C}_{(3)}=-\frac{L^{3}}{g_{5} z^{4}} d t \wedge d x_{1} \wedge d x_{2}, \quad B_{(2)}=\frac{L^{2}}{q z^{2}} d t \wedge d y,
\end{aligned}
$$

where $g_{5} \equiv \frac{g_{a}}{L^{5 / 2}}$ is the $5 d$ string coupling constant. The $g_{a}$ is 10 -dimensional coupling. The corresponding $5 d$ effective action can be presented as

$$
S_{5 d} \sim \int\left[e^{-2 \phi_{5}}\left(* R+4\left(d \phi_{5}\right)^{2}-\frac{1}{2}\left(d B_{(2)}\right)^{2}+* \frac{20}{L^{2}}\right)-\frac{\bar{m}^{2}}{2}\left(B_{(2)}\right)^{2}-\frac{1}{2}\left(d \bar{C}_{(3)}\right)^{2}-* \frac{\bar{m}^{2}}{2}\right]
$$

where the parameters are related as $\bar{m}=L^{\frac{5}{2}} m \equiv \frac{1}{g_{5} L}$. The $B$ field is explicitly massive. One may however rewrite the above action in terms of an axion field after the Hodge duality, $* d C_{(3)}=d C_{(0)}$. The background (3.11) is an exact solution of the action (3.12) which has two cosmological constant terms of opposite signs. 


\subsection{The 4d Proca model}

After explicit compactification on $S^{1} \times S^{5}$ the 10-dimensional background (3.1) reduces to the following 4-dimensional $\mathrm{Lif}_{4}^{(2)}$ solution

$$
\begin{aligned}
& d s^{2}=\bar{L}^{2}\left(-\frac{d t^{2}}{z^{4}}+\frac{d x_{1}^{2}+d x_{2}^{2}}{z^{2}}+\frac{d z^{2}}{z^{2}}\right) \\
& e^{\phi_{4}}=g_{4}, \quad \mathcal{A}_{(1)}=-\frac{L}{z^{2}} d t
\end{aligned}
$$

where the curvature radius $\bar{L} \equiv \frac{L}{g_{4}}$ and $g_{4} \equiv \frac{g_{a} \sqrt{q}}{L^{3}}$ is the $4 d$ string coupling constant. The dilaton has got constant background value. This background is a solution of the following Einstein-Proca effective action, which directly follows from $S^{1} \times S^{5}$ compactification of the Romans theory,

$$
S_{4 d} \sim \int\left[* R-2\left(d \phi_{4}\right)^{2}-\frac{e^{-2 \phi_{4}}}{2}\left(d \mathcal{A}_{(1)}\right)^{2}-\frac{m_{4}^{2} e^{2 \phi_{4}}}{2}\left(\mathcal{A}_{(1)}\right)^{2}+* \frac{20 e^{2 \phi_{4}}}{L^{2}}-* \frac{5 m_{4}^{2} e^{4 \phi_{4}}}{2}\right]
$$

where new mass parameter $m_{4}=\frac{2}{g_{4} L}$. The action includes a Proca field (descending from the winding modes of the massive B-field in (3.1)). If we plug in the constant value of the dilaton $g_{4}$, the action (3.14) further simplifies to

$$
S_{4 d} \sim \int\left[* R-\frac{1}{2 g_{4}^{2}}\left(d \mathcal{A}_{(1)}\right)^{2}-\frac{2}{L^{2}}\left(\mathcal{A}_{(1)}\right)^{2}+* \frac{10 g_{4}^{2}}{L^{2}}\right] .
$$

We remind that the cosmological constant and Proca mass are very precisely related in the action so as to admit Lifshitz $a=2$ vacua (3.13). However the action (3.15) cannot have Schrödinger $a=3$ spacetime as solutions. Instead another Einstein-Proca action with a different cosmological constant, that follows from $S^{5}$ compactification of the fields in (2.4), will allow $S c h_{4}^{(3)}$ solutions, see for the details [23, 24]. From this example we realise that, although $4 d$ actions differ in only the value of cosmological constants, but their $10 d$ solutions have entirely different matter field contents. These massive gauge-gravity field models have been studied as effective gravity models describing holographic superconductor phenomena on 3 -dimensional boundary $[4,5,7,8]$. We shall provide alternative $4 d$ Lifshitz models which have two Maxwell potentials and they are obtainable by exploiting massive T-duality in the forthcoming sections.

\section{$4 \quad T^{2}$ dual of $(F 1, D 2, D 8)$ system}

The 8-dimensional type II supergravity has a T-duality group $\mathrm{SL}(2, R) \times \mathrm{SL}(2, R)$ including the massive version of supergravity [39]. Under this massive duality symmetry the mass parameter $m$ (or dual 10-form $G_{(10)}$ flux) in Romans theory compactified on $T^{2}$ gets mapped into $G_{(2)}$-flux (along 2-torus) in ordinary type-IIA compactified on $T^{2}$. Using this massive duality we would like to map Lif $_{4} \times R^{1} \times S^{5}$ vacua (3.1) into the $G_{(2)}$-flux vacua of ordinary type IIA theory. 
Let us choose $x_{1}$ and $x_{2}$ to be the coordinates along $T^{2}$. The RR 2-form flux corresponds to having a nonvanishing constant magnetic component

$$
G_{f l u x} \equiv m d x_{1} \wedge d x_{2}=\frac{2}{g_{a} L} d x_{1} \wedge d x_{2}
$$

filling the entire $T^{2}$. (Note we must take mass $m$ exactly equal to $\frac{2}{g_{a} L}$ as it is fixed earlier.) Using the duality map worked out in [39], we can now write down corresponding vacua of ordinary type IIA theory,

$$
\begin{aligned}
d s^{2} & =L^{2}\left(-\frac{d t^{2}}{z^{4}}+\frac{z^{2}}{L^{4}}\left(d x_{1}^{2}+d x_{2}^{2}\right)+\frac{d z^{2}}{z^{2}}+\frac{d y^{2}}{q^{2}}+d \Omega_{5}^{2}\right), \\
e^{\phi} & =\frac{z^{2}}{L^{2}} g_{a}, \quad G_{(2)}=\frac{4 L^{3}}{z^{5} g_{a}} d z \wedge d t+G_{\text {flux }}, \\
B_{(2)} & =\frac{L^{2}}{q z^{2}} d t \wedge d y,
\end{aligned}
$$

Thus it could see that corresponding RR 1-form gauge field has a most general form

$$
C_{(1)}=C_{0} d t+C_{1} d x^{1}+C_{2} d x^{2}, \quad C_{0}=-\frac{L^{3}}{g_{a} z^{4}}, \quad C_{1}=-\frac{1}{g_{a} L} x_{2}, \quad C_{2}=\frac{1}{g_{a} L} x_{1}
$$

which has both electric as well as magnetic components. However no $C_{(3)}$ background is present, that is because after the duality all D2 branes (of massive-IIA) now reapper as D0 branes in (4.2). The $B$ field being massless, as it is in ordinary type IIA, however interacts (or gets nested) with nontrivial $G_{(2)}$ flux of D6-branes in the effective action. (Note all D8 branes morphe into D6 branes after massive duality on $T^{2}$ ). The $B$ equation of motion has a contributions from the interaction term in 4-form field strength $G_{(4)}=d C_{(3)}+G_{(2)} \wedge B_{(2)}$ in type IIA, which for the above background (4.2) contributes a term like $\sim \frac{1}{L^{2}}\left(B_{t y}\right)^{2}$ in the action due to nonvanishing $G_{(2)}$ flux. The equation of motion of $C_{(3)}$ is trivially satisfied for $C_{(3)}=0$. Thus $G_{(2)}$ has got both electric (D0) and magnetic (D6) components all aligned along noncompact directions in $5 d$ spacetime in the above. Thus the Lifshitz background (4.2) essentially represents $(F 1, D 0, D 6)$ bound state in ordinary type IIA. The DO branes have reappeared back and F-strings are now massless. This maybe called as un-higgsing phenomenon carried out by massive duality on $T^{2}$.

\section{$5 \quad$ Lower dimensional models}

\subsection{4d Lifshitz theory and a quantum Hall system}

Let us now consider the compact case where $y \sim y+2 \pi R_{y}$ in (4.2). The D6-branes will wrap around $S^{1} \times S^{5}$ completely and their $G_{2}$ flux would fill entire $x_{1}-x_{2}$ plane. The massless F1-strings will be wrapping around $y$ circle. The $4 d$ string coupling goes to vanishing value in the UV (as $z \rightarrow 0)$. Hence these vacua after compactification give rise to $\widetilde{L i f}(2)$ vacua 
(in string metric)

$$
\begin{aligned}
d s^{2} & =-\frac{L^{2} d t^{2}}{z^{4}}+\frac{z^{2}}{L^{2}}\left(d x_{1}^{2}+d x_{2}^{2}\right)+\frac{L^{2} d z^{2}}{z^{2}} \\
e^{\phi_{4}} & =g_{a} \frac{\sqrt{q}}{L^{3}} \frac{z^{2}}{L^{2}}, \quad C_{(1)}=\frac{1}{g_{a}}\left(-\frac{L^{3}}{z^{4}} d t-\frac{x_{2}}{L} d x_{1}+\frac{x_{1}}{L} d x_{2}\right), \\
\mathcal{A}_{(1)} & =\frac{-L^{2}}{q z^{2}} d t
\end{aligned}
$$

with two distinct Maxwellian gauge fields $\mathcal{A}$ and $C$. The scaling property of the solution (5.1) under the $z \rightarrow \lambda z$ is

$$
\begin{aligned}
& t \rightarrow \lambda^{2} t, \quad x_{1} \rightarrow \lambda^{-1} x_{1}, \quad x_{2} \rightarrow \lambda^{-1} x_{2}, \\
& g_{\mu \nu} \rightarrow g_{\mu \nu}, \quad e^{\phi} \rightarrow \lambda^{2} e^{\phi}, \quad C \rightarrow \lambda^{-2} C, \quad \mathcal{A} \rightarrow \mathcal{A}
\end{aligned}
$$

It has a dynamical exponent of time as $a=2$, but crucially has 'negative' scaling exponent $\left(a_{x}=-1\right)$ for spatial directions $x_{1}$ and $x_{2}$. It is quite plausible because D0 branes are delocalised over $x_{1}, x_{2}$ plane as well as there is nontrivial magnetic flux $G_{12}$. (The negative scaling exponent of spatial coordinates is usually associated with negative pressure along those direction in the CFT. $)^{3}$

The background (5.1) is unique Lifshitz vacuum in the sense that it describes two 'electrically' charged objects interacting with a magnetic flux. It can be checked that the Lifshitz vacua (5.1) is indeed a solution of following massless $4 d$ effective action

$$
S_{4 d} \sim \int\left[e^{-2 \phi_{4}}\left(* R+4\left(d \phi_{4}\right)^{2}-\frac{q^{2}}{2 L^{2}}\left(d \mathcal{A}_{(1)}\right)^{2}+* \frac{20}{L^{2}}\right)-\frac{L^{6}}{2 q} G_{(2)}^{2}-\frac{q L^{4}}{2}\left(\mathcal{A}_{(1)} \wedge G_{(2)}\right)^{2}\right]
$$

where $\phi_{4}$ is 4-dimensional dilaton field and field strength $G_{(2)}=d C_{(1)}$. Note that the scaling of the fields in (5.2) gives rise to following property of the action:

$$
S_{4 d} \rightarrow \frac{S_{4 d}}{\lambda^{4}}
$$

One thing to observe is that there is no mass term for gauge fields in the action (5.3). However two Maxwell fields in the action have Chern-Simons like interaction between them. Hence they give rise to two types of charged objects with a Chern-Simons like interaction $(\mathcal{A} \wedge d C)^{2}$ between them. The boundary Lifshitz theory lives over 2-dimensional spatial plane. By constant scaling of the fields the action (5.3) can be brought to a canonical form

$$
S_{4 d} \sim \int\left[* R-2\left(d \phi_{4}\right)^{2}-\frac{e^{-2 \phi_{4}}}{2}\left(d \mathcal{A}_{(1)}\right)^{2}+* \frac{20}{L^{2}} e^{2 \phi_{4}}-\frac{1}{2}\left(d C_{(1)}\right)^{2}-\frac{e^{-2 \phi_{4}}}{2}\left(\mathcal{A}_{(1)} \wedge d C_{(1)}\right)^{2}\right]
$$

\footnotetext{
${ }^{3}$ The nonrelativisitic phenomenon is expressed by the hamiltonian $H=\frac{(\vec{p})^{2}}{2 m}+\cdots$, and the scaling property (5.2) implies that the quasi-particle masses must scale as $m \rightarrow \lambda^{4} m$. Hence the particle mass (number) increases when we probe bigger length scales (or at low energy). It should be plausible for a quantum system exhibiting negative dynamical exponent for spatial coordinates.
} 
and corresponding $4 d$ solution also can be written as $^{4}$

$$
\begin{aligned}
d s^{2} & =L^{2}\left(-\frac{d t^{2}}{z^{4}}+\frac{z^{2}}{L^{4}}\left(d x_{1}^{2}+d x_{2}^{2}\right)+\frac{d z^{2}}{z^{2}}\right), \\
e^{\phi_{4}} & =g_{0} \frac{z^{2}}{L^{2}}, \quad C_{(1)}=\frac{1}{g_{0}}\left(-\frac{L^{3}}{z^{4}} d t-\frac{x_{2}}{L} d x_{1}+\frac{x_{1}}{L} d x_{2}\right), \\
\mathcal{A}_{(1)} & =-\frac{L}{z^{2}} d t,
\end{aligned}
$$

where $g_{0}\left(\equiv g_{a} \frac{\sqrt{q}}{L^{3}}\right)$ is $4 d$ coupling constant. The parameter $q$ has altogether disappeared from the solutions (5.6) and it only determines the coupling $g_{0}$. Since the dilaton runs towards strong coupling in the IR, this solution gives valid CFT descrition only in the $z<L$ (near UV region). In the UV region the Lifshitz theory becomes almost free.

It is tempting to holographically relate the bulk theory (5.5) to some known nonrelativistic condensed matter phenomenon in a plane on the boundary, involving two distinct (electrical) charges interacting in presence of constant magnetic field (or current), somewhat like in quantum Hall effect. There are no mass terms for gauge fields hence the solution (5.6) cannot describe superconducting or higgs phase. For the bulk solution (5.6), the gauge interaction terms in the action (5.5) is very crucial, but it is of rather unusual type to motivate. However it is not uncommon for such tensorial interactions to arise in bulk gravity (string) theory. The massless gauge-gravity action (5.5) certainly represents a new critical phenomenon, but it is also related via 'massive' T-duality to another action having massive gauge fields (3.15) and describing superconducting phenomenon $[4,7,8]$.

Next the gauge field $\mathcal{A}$ specially couples to the dilaton hence it is distinct as compared to the other gauge field $C$ which is totally decoupled from dilaton in (5.5). The former may thus have its origin in the Hall (excitaions) carriers. As we understand from the quantum Hall effect that, there is a constant magnetic field uniformally spread over a plane alongwith an electric field (EMF) applied in one (say $x_{1}$ ) direction of the plane. Consequently a quantized Hall voltage (current) gets generated along another (here $x_{2}$ ) direction of the planar system. From this analogy we understand that $\mathcal{A}_{0}$ can possibly be the source of Hall charges. In holography, the boundary value of the time-component of bulk gauge field represents charge sources in boundary. Furthermore, $\mathcal{A}_{0}$ is essentially a component of string $B$-field (wrapped on $y$ circle). Since $L^{4} \sim O(N) \gg 1$ and $L$ is an important overall parameter. We find that the two gauge potentials behave as

$$
\mathcal{A}_{0} \sim O\left(N^{\frac{1}{4}}\right), \quad C_{0} \sim O\left(N^{\frac{3}{4}}\right)
$$

Thus their respective carrier concentrations would have $O\left(N^{\frac{1}{2}}\right)$ difference. However if we compare respective Lorentz invariants in the action involving electric and magnetic fields,

\footnotetext{
${ }^{4}$ The $4 d$ string metric has a constant negative curvature. It does not include curvature singularity.
} 
then

$$
\begin{aligned}
e^{-2 \phi_{4}}\left(\partial_{z} \mathcal{A}_{0}\right)^{2} & \sim-\frac{4 g_{0}^{2}}{L^{6}} z^{4}, \\
\left(\partial_{z} C_{0}\right)^{2} & \sim-\frac{16 g_{0}^{2}}{L^{6}} z^{4}, \\
\left(\partial_{i} C_{j}\right)^{2} & \sim \frac{4 g_{0}^{2}}{L^{6}} z^{4} .
\end{aligned}
$$

It shows that, though the electric field contribution of $(\partial \mathcal{A})^{2}$ is comparatively weaker by a factor of $1 / N$, but in the lagrangian (5.5) all gauge terms contribute with equal strengths due to varied dilatonic couplings. These on-shell quantities do not diverge near the boundary. Furthermore, in the bulk solution (5.6) we can in fact choose a gauge such that $C_{0}, C_{1}$ components are only nonvanishing alongwith $\mathcal{A}_{0}$. This implies existence of two independent sources of charge (EMF) and an indepedent electric current in the boundary theory.

\subsection{Alternative vacua with negative dynamical exponent}

It is obvious that the action (5.5) would allow following Lifshitz vacua where dynamical exponent of time is instead negative while two spatial coordinates scale positively. Let us define a new holographic coordinate as

$$
u^{2}=\frac{L^{4}}{z^{2}}
$$

and by scaling $t \rightarrow L^{4} t$, we obtain from (5.6) a new kind of situation described by

$$
\begin{aligned}
d s^{2} & =L^{2}\left(-u^{4} d t^{2}+\frac{1}{u^{2}}\left(d x_{1}^{2}+d x_{2}^{2}\right)+\frac{d u^{2}}{u^{2}}\right), \\
e^{\phi_{4}} & =g_{0} \frac{L^{2}}{u^{2}}, \quad C_{(1)}=\frac{1}{g_{0}}\left(-\frac{u^{4}}{L} d t-\frac{x_{2}}{L} d x_{1}+\frac{x_{1}}{L} d x_{2}\right), \\
\mathcal{A}_{(1)} & =-u^{2} L d t,
\end{aligned}
$$

which is valid in the $u>L$ (IR) region. This new looking solution (5.10) has negative dynamical exponent for time $(a=-2)$, such that

$$
\begin{aligned}
& u \rightarrow \lambda u, \quad t \rightarrow \lambda^{-2} t, \quad x_{1} \rightarrow \lambda x_{1}, \quad x_{2} \rightarrow \lambda x_{2}, \\
& g_{\mu \nu} \rightarrow g_{\mu \nu}, \quad e^{\phi_{4}} \rightarrow \lambda^{-2} e^{\phi_{4}}, \quad C \rightarrow \lambda^{2} C, \quad \mathcal{A} \rightarrow \mathcal{A} .
\end{aligned}
$$

Note that the string coupling tends to blow up at shorter length scales (UV), while at longer (IR) scales it becomes almost a free theory. Thus the Lifshitz background (5.10) is suitable for describing a low energy Lifshitz theory at large length scales $u>L$. (While at shorter scales, $z<L$, the previous background (5.6) is more suitable.) In the present case the boundary field theory may actually describe electrodynamics because coupling remains weaker at longer distances. ${ }^{5}$ Once again there are two kinds of (electric) charges interacting with a constant magnetic field such as in quantum Hall systems near criticality.

\footnotetext{
${ }^{5}$ The nonrelativisitic phenomenon is expressed by the hamiltonian $H=\frac{(\vec{p})^{2}}{2 m}+\cdots$, and the scaling property (5.11) implies that the quasi-particle masses must scale as $m \rightarrow m / \lambda^{4}$. That is the quasi particle mass (number) get reduced when probed at large length scales (or with low energy) which appears to be plausible in a quantum system exhibiting negative dynamical exponent (for time) and having running coupling.
} 


\section{$5.35 d$ vortex model: $B \wedge G$ interaction}

A $5 d$ effective action can be obtained by compactification of type IIA theory on the product spacetime like $\mathcal{M}_{5} \times S^{5}$. We allow fields to have dependence only on $\mathcal{M}_{5}$ coordinates, such as we obtained in the background (4.2). Upon consistent truncations, and keeping only the relevant field content describing the equation (4.2), we get to following $5 d$ action

$$
S_{5} \sim \int\left[e^{-2 \phi_{5}}\left(* R+4\left(d \phi_{5}\right)^{2}-\frac{1}{2} H_{(3)}^{2}+* \frac{20}{L^{2}}\right)-\frac{1}{2} G_{(2)}^{2}-\frac{1}{2}\left(B_{(2)} \wedge G_{(2)}\right)^{2}\right]
$$

The action has second rank tensor with field strength $H_{(3)}=d B_{(2)}$ and a vector field with $G_{(2)}=d C_{(1)}$, both interacting through a $B \wedge G$ like coupling. This interaction term will be important when string-like excitations (or flux tubes) couple with constant magnetic field in a transverse $2 d$ plane in $4 d$ CFT. As an example, the equation of motion of the action (5.12) are immediately solved by the following solution

$$
\begin{aligned}
d s^{2} & =-\frac{L^{2} d t^{2}}{z^{4}}+\frac{L^{2} d y^{2}}{q^{2}}+\frac{z^{2}}{L^{2}}\left(d x_{1}^{2}+d x_{2}^{2}\right)+\frac{L^{2} d z^{2}}{z^{2}}, \\
e^{\phi_{5}} & =g_{0} \frac{z^{2}}{L^{2}}, \quad G_{(2)}=\frac{4 L^{3}}{g_{0} z^{5}} d z \wedge d t+\frac{1}{g_{0} L} d x_{1} \wedge d x_{2}, \\
B_{(2)} & =-\frac{L^{2}}{q z^{2}} d t \wedge d y,
\end{aligned}
$$

where $g_{0}=g_{a} / \sqrt{L^{5}}$ is $5 d$ coupling constant and $q$ is arbitrary having dimensions of length. The $5 d$ spacetime has constant negative curvature. The coupling remains weak in the UV region. The solution represents an uniform string like excitation extended in $y$ direction and a constant magnetic $G$-flux $\sim \frac{1}{g_{0} L}$ in the transverse $x_{1}-x_{2}$ plane.

The above solution (5.13) is however well behaved only in the UV region where $z<L$. But, by using the transformation (5.9) one can transform it into the IR region $(u>L)$

$$
\begin{aligned}
d s^{2} & =L^{2}\left(-\frac{d t^{2}}{u^{-4}}+\frac{d x_{1}^{2}+d x_{2}^{2}}{u^{2}}+\frac{d u^{2}}{u^{2}}+\frac{d y^{2}}{q^{2}}\right) \\
e^{\phi_{5}} & =g_{0} \frac{L^{2}}{u^{2}}, \quad G_{(2)}=\frac{4 u^{3}}{g_{0} L} d u \wedge d t+\frac{1}{g_{0} L} d x_{1} \wedge d x_{2}, \\
B_{(2)} & =-\frac{L^{2} u^{2}}{q} d t \wedge d y,
\end{aligned}
$$

where coupling remains weaker in the deep IR region. The dynamical exponent of time is given by $a=-2$. The charge sources are also present because the time-component $C_{0}$ is nontrivial. There is a constant magnetic background too, but there are also extended string (vortex) like objects present which couple to $B_{0 y}$. It would be worthwhile to do a detailed analysis of the nonrelativistic CFT on the boundary. We hope to come back to this topic in a future investigation.

\section{6 (W, M2) brane system}

The (F1,D2,D8) system (3.1) cannot be uplifted to M-theory at strong coupling as it is a solution of Romans theory for which we do not have 11-dimensional interpretation. Also 
because $L \sim \frac{1}{m g_{a} l_{s}} \rightarrow 0$ at strong coupling the spacetime becomes highly curved. On the other hand, we obtained (F1,D0,D6) in eq.(4.2) as the solution of ordinary type IIA supergravity. For the massless type IIA theory there exists an M-theory description [42]. For the vacua (4.2) the string coupling becomes strong in the IR region $(z \rightarrow \infty)$ which usually is the case for $\mathrm{CFT}_{3}$ (in relativistic cases also) [43]. At strong coupling a natural explanation of the theory should be found by studying these solutions in M-theory (with $\left.R_{11}=g_{a}^{2 / 3} l_{p}, l_{p}=g_{a}^{-1 / 3} l_{s}\right)$

$$
d s_{11 d}^{2}=e^{\frac{4 \phi}{3}}\left(d x_{11}+A\right)^{2}+e^{\frac{-2 \phi}{3}} d s_{10 d}^{2}
$$

where $x_{11} \sim x_{11}+2 \pi R_{11}$ is the eleventh coordinate. Correspondingly 11-dimensional supergravity vacua can be described as (setting $L=1$ for simplicity)

$$
\begin{aligned}
d s_{11 d}^{2} & =\frac{1}{z^{4 / 3}}\left(-\frac{d t^{2}}{z^{4}}+z^{2}\left(d x_{1}^{2}+d x_{2}^{2}\right)+\frac{d z^{2}}{z^{2}}+z^{4}\left(d x_{11}+\omega\right)^{2}+\frac{1}{w^{2}} d \psi^{2}+d \Omega_{5}^{2}\right), \\
C_{(3)} & =\frac{1}{w z^{2}} d t \wedge d x_{11} \wedge d \psi,
\end{aligned}
$$

where fiber 1 -form is

$$
\omega_{(1)}=-z^{-4} d t-x_{2} d x_{1}+x_{1} d x_{2} .
$$

The background in (6.1) represents a conformal $L i f_{5}^{(2)} \times S_{w}^{1} \times S^{5}$ vacua in M-theory which includes circle $x_{11}$ fibered over a Lif $_{4}$ base geometry: $-\frac{d t^{2}}{z^{4}}+z^{2}\left(d x_{1}^{2}+d x_{2}^{2}\right)+\frac{d z^{2}}{z^{2}}$. The fiber 1 -form is special in that it has both time as well as magnetic components. The magnetic components can be thought off as an uplift of D6-branes in (4.2). (Note D6 branes will have only geometric interpretation when lifted to M-theory where magnetic type IIA 1form becomes the fiber along M-theory circle.). The M2-branes wrap $\left(x_{11}, \psi\right)$ completely. There is large anisotropy along $x_{1}, x_{2}, x_{11}$ directions of the Lifshitz spacetime;

$$
t \rightarrow \lambda^{2} t, \quad x_{1} \rightarrow \lambda^{-1} x_{1}, \quad x_{2} \rightarrow \lambda^{-1} x_{2}, \quad x_{11} \rightarrow \lambda^{-2} x_{11}
$$

under which the metric conformally scales as $g_{\mu \nu} \rightarrow \lambda^{-4 / 3} g_{\mu \nu}$ while the 3 -form $C \rightarrow \lambda^{-2} C$.

\section{Summary}

We have shown that for the Romans' type IIA supergravity the $(F 1, D 2, D 8)$ brane configuration gives rise to the Lifshitz vacua $\operatorname{Lif}_{4}^{(2)} \times R^{1} \times S^{5}$. For this Lifshitz solution the fundamental strings have to be massive with $m=\frac{1}{g_{a} L l_{s}}$, and such that $L \gg 1$ and $g_{a} \ll 1$. A compactification of the massive type IIA theory on $S^{1}$ relates 9-dimensional $L i f_{4}^{(2)} \times S^{5}$ background with corresponding Scherk-Schwarz reduced type IIB Lifshitz vacua compactified on a dual circle. This duality map is known as 'massive' T-duality [33]. It has been known in litrature that $\operatorname{Lif}_{4}^{(2)} \times S^{1} \times S^{5}$ spacetime is constituted by axion-flux $(W, D 3, D 7)$ system in type IIB string theory. The presence of D7-branes primarily requires constant axion flux switched on along $S^{1}$ in these solutions. None of these solutions preserve any supersymmetry. 
Further, using 'massive' T-duality symmetry in eight dimensions, we have related the Lifshitz vacua in Romans theory to yet another $\widetilde{\operatorname{Lif}_{4}^{(2)}} \times R^{1} \times S^{5}$ vacua which is constituted by $(F 1, D 0, D 6)$ brane system of ordinary type IIA theory. But these latter vacua have negative dynamical exponents along two CFT directions. The interesting observation is that this $(F 1, D 0, D 6)$ solution upon exlicit compactification along $S^{1} \times S^{5}$ gives rise to $4 d$ Lifshitz vacua with two distinct types of electric charges supported by a constant magnetic flux (or current), all entirely living over $2 d$ plane at the boundary. We speculate that this may represent phenomena akin to quantum Hall systems. We also have obtained M-theory uplift of the $a=2$ Lifshitz solutions.

\section{Acknowledgments}

I am thankful to the ICTP, Trieste for the associateship support and the ASC-LMU, Munich for kind hospitality where parts of this work are carried out.

Open Access. This article is distributed under the terms of the Creative Commons Attribution License (CC-BY 4.0), which permits any use, distribution and reproduction in any medium, provided the original author(s) and source are credited.

\section{References}

[1] J.M. Maldacena, The large-N limit of superconformal field theories and supergravity, Int. J. Theor. Phys. 38 (1999) 1113 [hep-th/9711200] [INSPIRE].

[2] S.S. Gubser, I.R. Klebanov and A.M. Polyakov, Gauge theory correlators from noncritical string theory, Phys. Lett. B 428 (1998) 105 [hep-th/9802109] [INSPIRE].

[3] E. Witten, Anti-de Sitter space and holography, Adv. Theor. Math. Phys. 2 (1998) 253 [hep-th/9802150] [INSPIRE].

[4] D.T. Son, Toward an AdS/cold atoms correspondence: a geometric realization of the Schrödinger symmetry, Phys. Rev. D 78 (2008) 046003 [arXiv: 0804.3972] [InSPIRE].

[5] K. Balasubramanian and J. McGreevy, Gravity duals for non-relativistic CFTs, Phys. Rev. Lett. 101 (2008) 061601 [arXiv:0804.4053] [inSPIRE].

[6] C.P. Herzog, M. Rangamani and S.F. Ross, Heating up Galilean holography, JHEP 11 (2008) 080 [arXiv:0807.1099] [INSPIRE].

[7] S.A. Hartnoll, C.P. Herzog and G.T. Horowitz, Holographic superconductors, JHEP 12 (2008) 015 [arXiv:0810.1563] [INSPIRE].

[8] S.A. Hartnoll, C.P. Herzog and G.T. Horowitz, Building a holographic superconductor, Phys. Rev. Lett. 101 (2008) 031601 [arXiv:0803.3295] [INSPIRE].

[9] J. Maldacena, D. Martelli and Y. Tachikawa, Comments on string theory backgrounds with non-relativistic conformal symmetry, JHEP 10 (2008) 072 [arXiv:0807.1100] [INSPIRE].

[10] F. Denef and S.A. Hartnoll, Landscape of superconducting membranes, Phys. Rev. D 79 (2009) 126008 [arXiv:0901.1160] [InSPIRE].

[11] A. Donos and J.P. Gauntlett, Supersymmetric solutions for non-relativistic holography, JHEP 03 (2009) 138 [arXiv:0901.0818] [INSPIRE]. 
[12] A. Donos and J.P. Gauntlett, Solutions of type IIB and D=11 supergravity with Schrödinger(z) symmetry, JHEP 07 (2009) 042 [arXiv: 0905.1098] [INSPIRE].

[13] A. Donos and J.P. Gauntlett, Schrödinger invariant solutions of type IIB with enhanced supersymmetry, JHEP 10 (2009) 073 [arXiv:0907.1761] [INSPIRE].

[14] C. Duval and P.A. Horvathy, Non-relativistic conformal symmetries and Newton-Cartan structures, J. Phys. A 42 (2009) 465206 [arXiv:0904.0531] [INSPIRE].

[15] A. Bagchi and R. Gopakumar, Galilean conformal algebras and AdS/CFT, JHEP 07 (2009) 037 [arXiv:0902.1385] [INSPIRE].

[16] J.P. Gauntlett, J. Sonner and T. Wiseman, Holographic superconductivity in M-theory, Phys. Rev. Lett. 103 (2009) 151601 [arXiv:0907.3796] [INSPIRE].

[17] S.S. Gubser, C.P. Herzog, S.S. Pufu and T. Tesileanu, Superconductors from superstrings, Phys. Rev. Lett. 103 (2009) 141601 [arXiv:0907.3510] [InSPIRE].

[18] C.R. Hagen, Scale and conformal transformations in Galilean-covariant field theory, Phys. Rev. D 5 (1972) 377 [inSPIRE].

[19] T. Mehen, I.W. Stewart and M.B. Wise, Conformal invariance for nonrelativistic field theory, Phys. Lett. B 474 (2000) 145 [hep-th/9910025] [INSPIRE].

[20] Y. Nishida and D.T. Son, Nonrelativistic conformal field theories, Phys. Rev. D 76 (2007) 086004 [arXiv:0706.3746] [INSPIRE].

[21] C. Duval, G.W. Gibbons and P. Horvathy, Celestial mechanics, conformal structures and gravitational waves, Phys. Rev. D 43 (1991) 3907 [hep-th/0512188] [INSPIRE].

[22] C. Duval, M. Hassaine and P.A. Horvathy, The geometry of Schrödinger symmetry in gravity background/non-relativistic CFT, Annals Phys. 324 (2009) 1158 [arXiv:0809.3128] [INSPIRE].

[23] H. Singh, Galilean anti-de-Sitter spacetime in Romans theory, Phys. Lett. B 682 (2009) 225 [arXiv:0909.1692] [INSPIRE].

[24] H. Singh, Galilean type IIA backgrounds and a map, Mod. Phys. Lett. A 26 (2011) 1443 [arXiv: 1007.0866] [INSPIRE].

[25] K. Balasubramanian and K. Narayan, Lifshitz spacetimes from AdS null and cosmological solutions, JHEP 08 (2010) 014 [arXiv: 1005.3291] [INSPIRE].

[26] H. Singh, Special limits and non-relativistic solutions, JHEP 12 (2010) 061 [arXiv: 1009.0651] [INSPIRE].

[27] H. Singh, Lifshitz/Schrödinger Dp-branes and dynamical exponents, JHEP 07 (2012) 082 [arXiv: 1202.6533] [INSPIRE].

[28] R. Gregory, S.L. Parameswaran, G. Tasinato and I. Zavala, Lifshitz solutions in supergravity and string theory, JHEP 12 (2010) 047 [arXiv: 1009.3445] [INSPIRE].

[29] L. Barclay, R. Gregory, S. Parameswaran, G. Tasinato and I. Zavala, Lifshitz black holes in IIA supergravity, JHEP 05 (2012) 122 [arXiv:1203.0576] [INSPIRE].

[30] M. Taylor, Lifshitz holography, Class. Quant. Grav. 33 (2016) 033001 [arXiv:1512.03554] [INSPIRE].

[31] L.J. Romans, Massive $N=2 a$ supergravity in ten-dimensions, Phys. Lett. B 169 (1986) 374 [INSPIRE]. 
[32] J. Polchinski, Dirichlet branes and Ramond-Ramond charges, Phys. Rev. Lett. 75 (1995) 4724 [hep-th/9510017] [INSPIRE].

[33] E. Bergshoeff, M. de Roo, M.B. Green, G. Papadopoulos and P.K. Townsend, Duality of type-II 7 branes and 8 branes, Nucl. Phys. B 470 (1996) 113 [hep-th/9601150] [InSPIRE].

[34] E. Witten, BPS bound states of D0-D6 and D0-D8 systems in a B field, JHEP 04 (2002) 012 [hep-th/0012054] [INSPIRE].

[35] M. Mihailescu, I.Y. Park and T.A. Tran, D-branes as solitons of an $N=1, D=10$ noncommutative gauge theory, Phys. Rev. D 64 (2001) 046006 [hep-th/0011079] [INSPIRE].

[36] A. Fujii, Y. Imaizumi and N. Ohta, Supersymmetry, spectrum and fate of D0-Dp systems with B field, Nucl. Phys. B 615 (2001) 61 [hep-th/0105079] [INSPIRE].

[37] C.M. Hull, Massive string theories from M-theory and F-theory, JHEP 11 (1998) 027 [hep-th/9811021] [INSPIRE].

[38] M. Haack, J. Louis and H. Singh, Massive type IIA theory on K3, JHEP 04 (2001) 040 [hep-th/0102110] [INSPIRE].

[39] H. Singh, Duality symmetric massive type II theories in $D=8$ and $D=6$, JHEP 04 (2002) 017 [hep-th/0109147] [INSPIRE].

[40] H. Singh, Note on $(D 6, D 8)$ bound state, massive duality and noncommutativity, Nucl. Phys. B 661 (2003) 394 [hep-th/0212103] [INSPIRE].

[41] J.C. Breckenridge, G. Michaud and R.C. Myers, More D-brane bound states, Phys. Rev. D 55 (1997) 6438 [hep-th/9611174] [INSPIRE].

[42] E. Witten, String theory dynamics in various dimensions, Nucl. Phys. B 443 (1995) 85 [hep-th/9503124] [INSPIRE].

[43] N. Itzhaki, J.M. Maldacena, J. Sonnenschein and S. Yankielowicz, Supergravity and the large- $N$ limit of theories with sixteen supercharges, Phys. Rev. D 58 (1998) 046004 [hep-th/9802042] [INSPIRE]. 